\title{
DCX Gene
}

National Cancer Institute

\section{Source}

National Cancer Institute. DCX Gene. NCI Thesaurus. Code C75320.

This gene is involved in neuronal migration. 